Proc. 14th. Inf. Conf. on Low Temp. Phys., North Holland Pub. Co., Amsterdam, 5, 102; 1975) to the recent LT14 conference in Helsinki-but he was careful to avoid alluding to any numbers. In particular, he did not make any mention of a value for $T_{\text {r }}$

Patton and Zaringhalam have now described a theory which, with one adjustable parameter, gives a reasonable description of the pressure dependence of $T_{c}$ for pure ${ }^{3} \mathrm{He}$. They fix this parameter by fitting their theory to the experimental results already obtained and then, together with a variety of other experimental information, use it to derive a value of $T_{\text {r }}$, for a ${ }^{3} \mathrm{He}-{ }^{4} \mathrm{He}$ solution. They conclude that, for a saturated solution of $8.85 \%{ }^{3} \mathrm{He}$ in ${ }^{4} \mathrm{He}$ under a pressure of $20 \mathrm{bar}, T_{\mathrm{r}}=0.9 \mathrm{mK}$. This result is some 10,000 times larger than an earlier prediction by $\emptyset$ stgaard (Phys. Lett., 94A, 433; 1974) of $\sim 10^{-4}$ $\mathrm{mK}$.

These calculated values of $T_{c}$ may be compared with $0.7 \mathrm{mK}$, the lowest temperature to which pure ${ }^{3} \mathrm{He}$ has so far been cooled. Making thermal contact to a ${ }^{3} \mathrm{He}-{ }^{4} \mathrm{He}$ solution is rather more difficult then to pure ${ }^{3} \mathrm{He}$ at these temperatures, however, so that cooling a solution below $0.9 \mathrm{mK}$ may not necessarily be quite within present experimental capabilities; but it certainly cannot be far removed.

Remembering the bitter experience of the theoretical $T_{\mathrm{c}}$ values for pure ${ }^{3} \mathrm{He}$, a certain level of scepticism is inevitable in relation to Patton and Zaringhalam's $0.9 \mathrm{mK}$. It is nonetheless encouraging, and we should not have to wait very long for their prediction to be put to the test in an actual experiment.

\section{No need for glycophorin?}

from a Correspondent

IT is a truism that any breakthrough in science, as in art, fashion or sport, quickly becomes a commonplace. Nowhere is this more obvious than in protein sequencing. However, although the complete sequencing of a protein no longer has, perhaps quite the same impact as previously, the sequencing of a membrane protein is still a relatively rare occurrence. This has been achieved recently for glycophorin, a major protein of the human erythrocyte membrane (Tomita and Marchesi, Proc. natn. Acad. Sci. U.S.A., 72, 2964-2968; 1975; see News and Views, 258, 478; 1975). Some of the sequence is familiar from earlier publications by Marchesi and his colleagues. In par- ticular the now famous sequence of 23 amino acids lacking charged residues and enriched in hydrophobic residues such as leucine, isoleucine and valine, is placed firmly towards the carboxyl terminal of the polypeptide chain. This sequence is thought to be responsible for integrating the protein into the hydrophobic environment of the membrane. A segment of the polypeptide chain at the carboxyl end is believed to protrude internally whereas the amino terminal sequence, bearing a large amount of carbohydrate, is on the outer side of the membrane.

The function of this quantitatively important constituent of the erythrocyte membrane is unknown. Some recent results suggest that it may be dispensed with altogether without undue effect on the survival either of the erythrocyte or of the person carrying these cells.

The starting point for this work was the identification of a rare genetic variant in humans which affects erythrocyte surface antigens. The $\mathrm{En}^{\mathrm{a}}$ antigen is present on the surface of almost all human erythrocytes except in some very rare individuals. Three families are known with this trait: two in Finland and one in England (Dainborough et al., Vox Sang., 17, 241-255; 1969; Furuhjelm, et al., Vox Sang., 24, 545$549 ; 1973)$. These individuals, who are clinically normal, also show unusually weak activity of another common erythrocyte antigenic system called MN. It has been known for many years that $\mathrm{MN}$ antigenicity is associated with glycophorin and this prompted Tanner and Anstee (Biochem. J., 153, 271-277; 1976) to look for this component in En(a-) erythrocytes. It turns out that the erythrocytes apparently lack glycophorin completely. Erythrocyte En(a-) membranes analysed by SDS polyacrylamide gel electrophoresis have normal peptide composition except for the loss of glycophorin and a minor glycoprotein species of slightly smaller molecular weight.

Tanner and Anstee then tried to label glycophorin in En(a-) erythrocytes with lactoperoxides. In normal erythrocytes, glycophorin and another membrane protein are readily iodinated by this means. In En(a-) erythrocytes however, only one membrane component was labelled and this was not glycophorin.

What these results imply, therefore, is that glycophorin itself is not absolutely required for the function of the erythrocyte. This is surprising on two counts; first, glycophorin is one of the two most abundant proteins of the erythrocyte membrane. Together with a component called protein 3 it accounts for almost half of the membrane proteins. Second, there is considerable evidence that glycophorin and protein
3 form a non-covalent complex in the membrane. It has been suggested (see Rothstein et al., Fedn Proc., 35, 3-10; 1976) that the complex plays a coordinated role in anion transport. Although protein 3 appears to be the more directly involved in anion transport, it will be interesting to see if the quite drastic changes in the composition of membrane protein of En(a-) erythrocytes result in any physiological effects on ion transport.

\section{Aspects of tumour biology}

from M. J. Bevan and I. S. Trowbridge

The Armand Hammer Symposium in Tumour Biology was held at The Salk Institute, San Diego in January 1976.

THE origin of the cancer cell and its interaction with the immune system were the main themes of the Symposium.

In the opening session, David Baltimore (Massachusetts Institute of Technology) and Charles Heidelberger (McArdle Laboratory, Madison) discussed viral and chemical oncogenesis respectively. Baltimore showed that the resistance to infection by $\mathrm{N}$-tropic or B-tropic murine leukaemia viruses conferred by the FV-1 locus is probably at the level of transcription implying that $\mathrm{FV}-1$ is a regulatory gene coding for a repressor. Viral DNA could be made and integrated into the genome of resistant host cells but unlike the case of sensitive host cells virtually no viral RNA was produced. $\mathrm{He}$ also described the in vitro transformation of murine bone marrow and foetal liver cells by Abelson Leukaemia virus and its quantitation by a focal assay. Whether the target cell for transformation is a pre-B cell or a more primitive stem cell and how many such cells are present in the various haemopoietic organs is uncertain. It seemed unlikely that a Bcell itself is the target as no immunoglobulin-bearing cells were transformed.

Heidelberger established that chemical carcinogens actually transform normal cells rather than select pre-existing malignant cells from within the population of "normal" cells in his in vitro fibroblast cultures, by showing that frequencies of transformation greater than $50 \%$ are possible. He also described evidence that chemical carcinogens do not cause transformation 